\title{
Article
}

\section{Accuracy and Repeatability Tests on HoloLens 2 and HTC Vive}

\author{
Inês Soares ${ }^{1,2}\left(\mathbb{D}\right.$, Ricardo B. Sousa ${ }^{1,2} \mathbb{D}$, Marcelo Petry ${ }^{2}(\mathbb{D})$ and António Paulo Moreira ${ }^{1,2}(\mathbb{D})$ \\ 1 Faculdade de Engenharia da Universidade do Porto (FEUP, R. Dr. Roberto Frias, 4200-465 Porto); \\ 2 INESC TEC - Institute for Systems and Computer Engineering, Technology and Science, Porto, Portugal. \\ * Correspondence: marcelo.petry@inesctec.pt
}

\begin{abstract}
Augmented and Virtual Reality have been experiencing a rapidly growth in recent years, but there is not still a deep knowledge on their capabilities and where they could be explored. In that sense, this paper presents a study on the accuracy and repeatability of the Microsoft's HoloLens 2 (Augmented Reality device) and HTC Vive (Virtual Reality device) using an OptiTrack system as ground truth. For the HoloLens 2, the method used was hand tracking, while in HTC Vive, the object tracked was the system's hand controller. A series of tests in different scenarios and situations were performed to explore what could influence the measures. The HTC Vive obtained results in the millimetre scale, while the HoloLens 2 revealed not so accurate measures (around 2 centimetres). Although the difference can seem to be considerable, the fact that HoloLens 2 was tracking the user's hand and not an inherit controller made a huge impact. The results were considered a significant step for the on going project of developing a human-robot interface to program by demonstration an industrial robot using Extended Reality, which shows great potential to succeed based on this data.
\end{abstract}

Keywords: Programming by Demonstration; Virtual Reality; Augmented Reality; Accuracy; Repeatability.

\section{Introduction}

Fang et al. [1] define Human-Robot Interaction (HRI) as "the process that conveys the human operators' intention and interprets the task descriptions into a sequence of robot motions complying with the robot capabilities and the working requirements". This interaction can also be defined as a situation where humans and robots work as a team in order to reach a common goal. Each application of HRI demands a different level of interaction, such level is identified depending on two principles [1]: autonomy degree of the robotic system and proximity of human and robot during operation.

Augmented Reality (AR), Virtual Reality (VR) and Mixed Reality (MR) are well-known technologies applied in different areas with different purposes. Game environments, educational contents, medicine are some of the applications. Augmented Reality is when virtual objects and information are overlaid on the real world. In Virtual Reality, in contrast to $\mathrm{AR}$, the users experience a world completely virtual, they are fully immersed in a simulated digital environment. The technology that integrates both AR and VR is called Mixed Reality, where digital and real objects co-exist and can interact with each other in real time [2]. Augmented Reality, Virtual Reality and Mixed Reality are well-known immersive technologies applied in different areas with different purposes. It has already been applied not only in games and entertainment, but also in industry (to train and display information to the operator) [3], healthcare (more specifically, in performing surgeries) [4], real state (layout scenarios) [5], and even grocery shopping (providing nutrition facts, health and wellness tips, and unhealthy selection warnings) [6].

Several devices may be used to allow these immersive environments. In terms of AR, the most used are Microsoft HoloLens, Magic Leap One, Epson Moverio and Google Glass. As For VR, the most used are HTC Vive, Oculus Quest, Valve Index and Sony PlayStation VR.

The purpose of this paper is to present the study made on the accuracy and repeatability of the Microsoft HoloLens 2 (AR) and HTC Vive (VR) devices, as well as the results obtained and suitable applications for each device in the robotic area. This study is integrated in a wider project that its final purpose is to develop an industrial prototype of a 
human machine interaction system through Extended Reality (XR), in which the objective is to enable an industrial operator without any programming experience to program a collaborative robot using XR devices. In this regard, this study provides a better understanding on the system's possibilities in terms of accuracy.

The remaining sections of this paper are organized as follows. Section 2 presents a literature review on the XR system's accuracy. Section 3 gives a detailed description of the methodology used for the tests execution, about the setup of each technology, the data synchronization methods, the data analysis process and the tests performed. Section 4 discloses the tests results and discusses their tenor and meaning, as well as some of the suitable applications for each device accordingly with the results obtained. Ultimately, section 5 presents the conclusion and suggestions for future work.

\section{Related Work}

Programming by demonstration can be a very important tool for an operator that does not have experience or knowledge with programming at all. This way they would be able to program the robot just by doing the task themselves and then the robot would do the same. Therefore, the study of the accuracy and repeatability of the XR systems are extremely important in order to be aware of their limitations.

Rudorfer et al. [7] presented an intuitive drag-and-drop programming method using Augmented Reality that could be performed by an operator without robot programming knowledge. In the implementation the devices used were the Microsoft HoloLens and the UR5 robot, integrated into a framework of web services. The main objective was the user to pick a recognized object and place it in a desired location, so that the robot could imitate. The robot started by acquiring the image, then it recognized the object and its pose. After recognizing all the objects, the objects were displayed in the AR device, overlaying the real ones. Then the robot control module extracts the initial and final coordinates of the desired locations and performs the referential transformations from the camera referential to the robot referential. Finally, the pick and place task could be executed. The results obtained by the prototype developed were successful but the robot's accuracy was unsatisfactory, mostly because they based the target position on the intersection of the user's gaze with the platform, which imposed a limitations to the system's accuracy.

Blankemeyer et al. [8] developed an AR application for HoloLens, and the prime objective was to enable operators to program a pick-and-place task in an industrial robot by linking real and virtual objects. For that, the user had to move the virtual object to the desired position. Next, the coordinates of the start and end points had to be transformed from the internal coordinate system of the HoloLens into the robot's base coordinate system. Finally, the trajectory planning was carried out directly by the robot controller. The results to the tests performed showed that the robot was able to complete the tasks with two components, but the researches assured that the same can be expected when adding more components. The accuracy and repeatability of the system were measured calculating the difference of the virtual and real objects, reaching values of 1 - 2 millimeters for accuracy and 3 - 5 millimeters for repeatability.

Spitzley and Karduna [9] studied the possibility to use the HTC Vive VR system for kinematic data collection by evaluating the accuracy of the position and orientation data. The study was made on the HTC Vive controller and tracker in comparison to a Polhemus Liberty magnetic tracking system sensor for angular and translational measurement error and signal drift. The mean errors obtained for both the hand controller and the tracker were below 3 millimeters for translational movements and the angular errors below 0.4 degrees. The authors concluded that the system would be suitable and reliable for kinematic data collection, nevertheless they admit that further investigation in necessary to accurately determine the system's capability for capturing high-volume movements. Furthermore, the Magnetic tracker is easily disturbed by metallic objects, which limits its use.

Niehorster et al. [10] also confirmed the HTC Vive system's high accuracy and precision validated through a quantitative test of the position and orientation. However, 
they concluded that the system's latency was low (around 22 milliseconds). Additionally, they found that when data was briefly lost, there was some variations in offsets, what led to the conclusion that, in that conditions, the HTC Vive system would not be suitable for scientific experiments that require accurate visual stimulation of self-motion.

The results obtained are promising and show that it is possible for an operator without any robot programming knowledge to program a collaborative robot by teaching it the desired task. One of the problems found in these researches was the lack of accuracy experienced by the robot on the picking and place task. In this study, repeatability and accuracy is be the focus of our methodology, comparing the results obtained from Extended Reality devices to a precision tracking system.

\section{Materials and Methods}

The prime objective of this work is to measure the accuracy and repeatability of the HoloLens 2 (HL2) and HTC Vive devices. For that it was used the OptiTrack system as a ground truth due to its submillimeter accuracy. Figure 1 represents an overview of the system developed for these tests, the data acquired by the Extended Reality devices and the OptiTrack system are sent to Robot Operating System (ROS), where they are synchronized. After that, in MATLAB, the data is analysed and the accuracy, repeatability and possible delays are calculated. Additionally, some plots to illustrate the results are drawn.

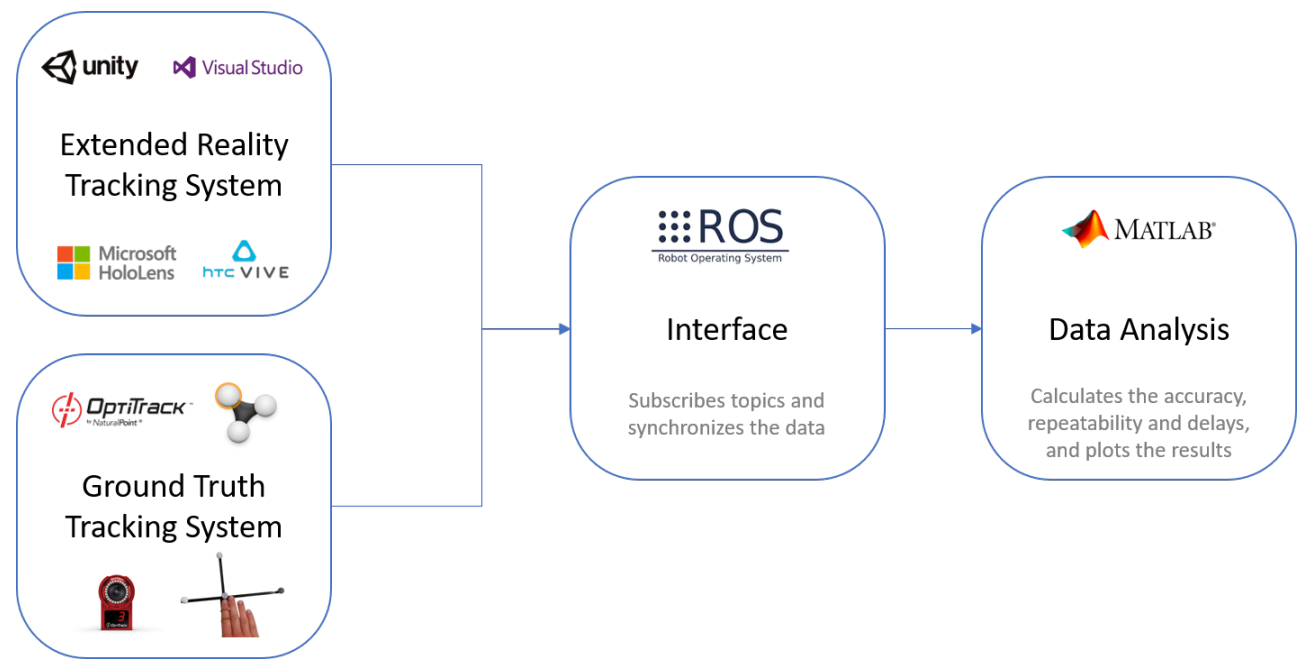

Figure 1. Overview of the system developed for the tests.

\subsection{Ground Truth Setup}

The OptiTrack system used was constituted by 6 Flex3 InfraRed cameras, 4 of them forming the vertices of a rectangle and the other 2 in the center of the biggest edges (Figure 2a). All the cameras were set up in the same plane (around 2.75 meters high) and covered a total area of 22 squared meters, but the used area to avoid occlusions was about 12 squared meters. The cameras were oriented to the center of the rectangle, which allowed a common ground between all the cameras and minimized the markers occlusions, and a full calibration using the OptiTrack software and hardware tool (Figure 2b) was performed. From that we obtained a mean 3D reprojection error of 0.791 millimeters.

After calibration, the ground plane was set using OptiTrack plane calibration tool (Figure 2c). This object had a shape of a squared triangle with three markers, one in each vertex, so that the system could identify the desired coordinate frame. One of the markers corresponds to the frame's origin and the other two to points in the $x$ and $z$ axis; this way the referential is defined taking into account that the y axis points upwards. 


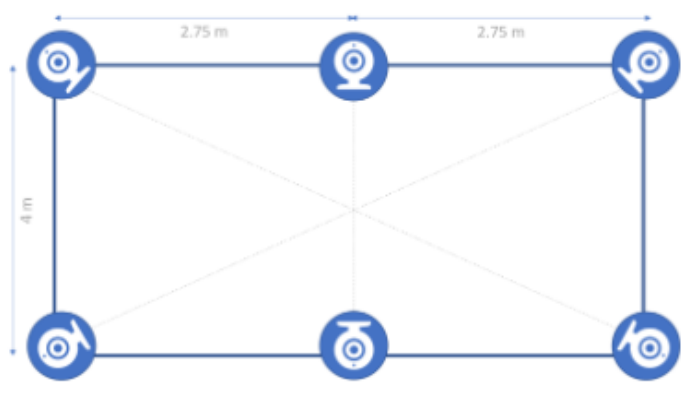

(a)

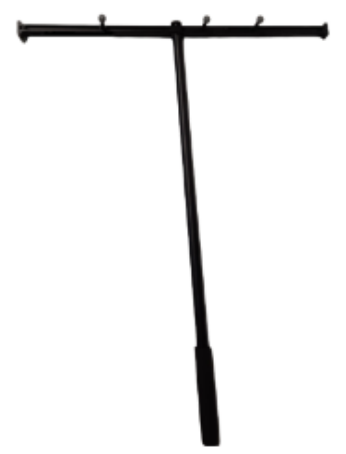

(b)

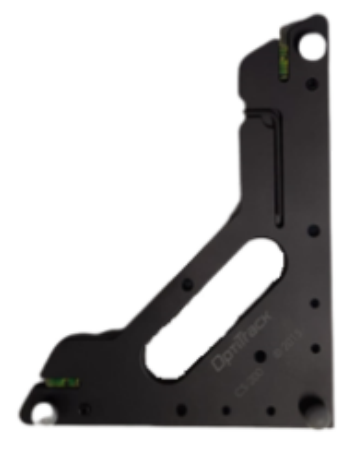

(c)

Figure 2. OptiTrack system: (a) OptiTrack setup. (b) Calibration tool. (c) Ground plane tool.

OptiTrack markers are small reflective spheres with 14 millimeter diameter (observable in Figure 2c). It is possible to acquire data from different types of assets: rigid body, skeleton and unlabelled markers. For the specific case of this study, it would be ideal to stream data from unlabelled markers, because it would only be necessary to place one marker on the user's hand. But this method is not reliable because the probability of the system losing track of it was very high, so the asset used was rigid body for being more accurate and trustworthy.

The system broadcasts the rigid body pose through the Virtual-Reality Peripheral Network (VRPN) streaming engine. To process the data, it was used the software framework Robot Operating System (ROS). The ROS package used to receive the data from OptiTrack was vrpn_client_ros. The message received from OptiTrack was in geometry_msgs/PoseStamped format, which contains a header with timestamp and the position and orientation of the rigid body. For this study the orientation was not considered.

\subsection{HoloLens 2 Setup}

As the position reference is a rigid body from OptiTrack system, it was necessary to print a 3D structure to hold the OptiTrack markers. The point considered for the HoloLens 2 measures was the tip of the index finger, therefore a rigid body was built so that its center was in the same place. There were some concerns in the construction of the rigid body, which are listed below:

- It should not be symmetrical because the OptiTrack system could be confused in some orientations;

- The minimum number of markers was three but it was opted to insert four, increasing the robustness of the rigid body detection, this way if one was hidden the system would continue tracking;

- The markers could not be close to each other, otherwise the system would not be able to track it properly. 
Taking all these limitations into consideration, the piece built had the shape of a cross, three of the markers formed a scalene triangle and the forth marker was placed in the center of the cross, Figure 3a. Additionally, the forth edge had the purpose of supporting the index finger (secured by two rubber bands), resulting in its tip touching the center marker of the rigid body. The resulting rigid body is represented in Figure $3 b$.

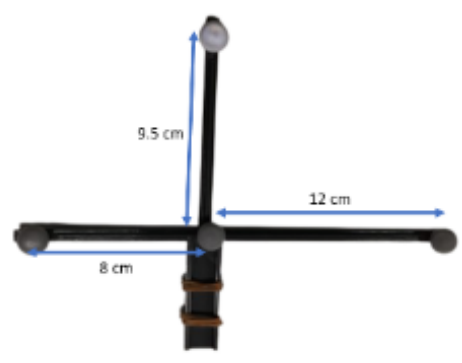

(a)

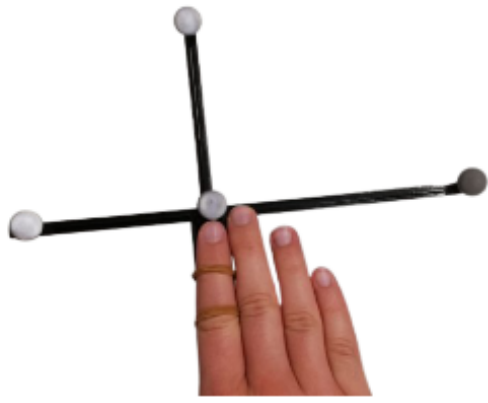

(b)

Figure 3. Rigid Body for tracking the user's index finger: (a) Rigid body dimensions. (b) Rigid body on the user's hand.

The HoloLens 2 defines automatically a coordinate system when the application is launched. So, in order to be able to compare directly the coordinates from OptiTrack and HL2, it was necessary to define a different referential to match the one from OptiTrack. Because the HL2 software does not allow to define a secondary referential, it was necessary to do some workarounds. The simplest way found was to place an object (a cube in this specific case) in the origin of the coordinate system and then calculate the hand coordinates in relation to that object. To define the cube's position and orientation, it was used the OptiTrack instrument for ground plane definition so that the coordinate systems would be exactly the same. To define the coordinate system in the HoloLens 2 application, it was used the user's right index finger tip, as it is explained below.

1. First, in order to define the cube's origin position, the user clicks on the interface button, suggesting that is ready to start defining the referential.

2. After clicking the button, the user has five seconds to place the right index finger tip at the OptiTrack coordinate system origin (Figure 4a). When that time is up, the system will save the coordinates where the right index finger tip is, as the cube's point of origin.

3. Then, the user clicks the button again and has five seconds to place the finger at the second point, which defines the point in the $X$ axis (Figure $4 b$ ).

4. The same happens with the third point (Figure 4c).

5. Lastly, the user clicks in the button again to confirm that the referential is correctly set and the cube appears at the defined origin with the specified orientation.

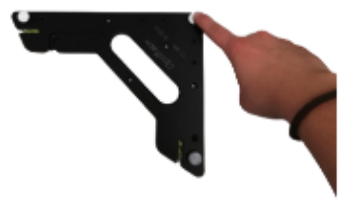

(a)

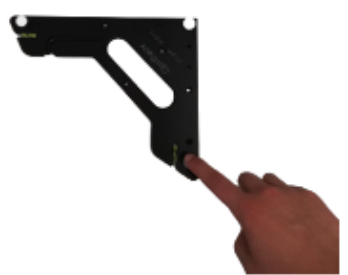

(b)

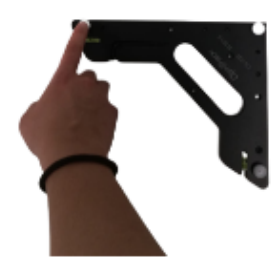

(c)

Figure 4. Referential definition on the HoloLens 2 application: (a) Step 1. (b) Step 2. (c) Step 3. 
The HL2 application to stream the hand position was built in Unity using the Microsoft's Mixed Reality Toolkit (MRTK ${ }^{1}$ ). In order to establish a connection with ROS, it was used the ROS\# $\#^{2}$ libraries. To be able to compare directly the message from HL2 and OptiTrack, the application streams to a ROS topic a PoseStamped message containing the timestamp and the position of the user's index finger tip, the orientation was set to zero because it was not used in this study.

\subsection{HTC Vive Setup}

The HTC Vive is a Virtual Reality headset that has a set of two hand controllers. The HTC Vive system has two base stations to capture the optical signals from the controllers so that they can be tracked. These base stations were positioned in opposite corners with a 5 meters distance from one another, and connected with a sync cable. So, instead of using the user's hand, one of the controllers was the tracking object to evaluate the accuracy and repeatability of the system. Furthermore, it was necessary to print a 3D structure, like in the HoloLens 2 application, that would represent the rigid body to track in OptiTrack. The main part of the piece was identical to the one described before, but instead of having a support to place the finger, it fitted in the controller's center hole.

The HTC Vive system has two base stations to capture the optical signals from the controllers so that they can be tracked. These base stations were positioned in opposite corners with a 5 meters distance from one another, and connected with a sync cable.

The origin of the controller's reference frame is represented in Figure 5. The orange ' $x$ ' in the figure illustrate the desired point to consider as the controller's center for more accurate measures. The rigid transformation between the frames was estimated using the controller's CAD model (0.0; 0.030986; 0.01946 meters) and implement trough MRTK Solver system.

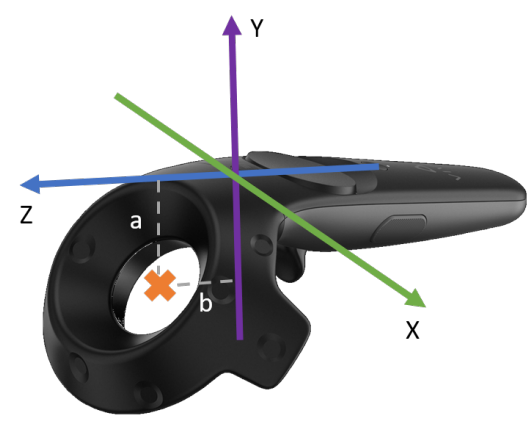

Figure 5. Coordinate system.

Similarly to HoloLens 2, the coordinate system definition was set also by three points using the OptiTrack plane calibration tool. However, instead of placing the index finger on the marker, it was placed the controller, more specifically the center of the controller's toroid pointing downwards. To make the measurements well grounded, it was printed a piece that fitted the toroid's cavity and in the center was an empty space that had the exact size to fit the reflective marker, Figure 6a.

\footnotetext{
https://github.com/microsoft/MixedRealityToolkit-Unity
}

2 https://github.com/siemens/ros-sharp 


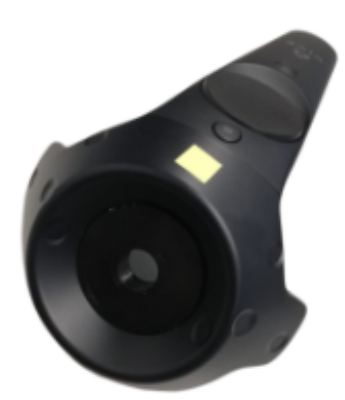

(a)

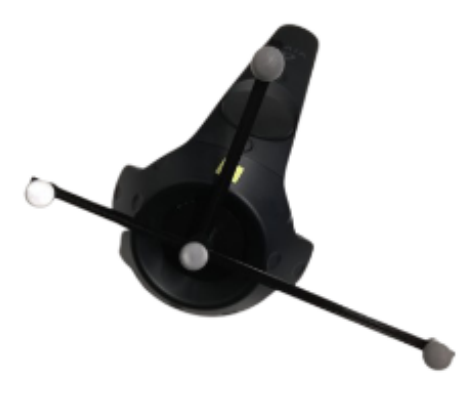

(b)

Figure 6. HTC Vive controller: (a) Calibration setup. (b) Tracking setup.

Figure 7 shows the sequence in which the application coordinate system is defined in HTC Vive, first defining the origin point, then the point in the $X$ axis and, finally, the point in the $\mathrm{Z}$ axis. The list below describes this process in more detail:

1. First, in order to define the cube's origin position, the user clicks with the controller on the interface button, suggesting that is ready to start defining the referential.

2. After clicking the button, the user has five seconds to place upside down the right controller at the OptiTrack coordinate system origin (Figure 7a). When that time is up, the system will save the coordinates where the controller is, as the cube's point of origin.

3. Then, the user clicks the button again and has five seconds to place the controller at the second point, which defines the point in the $\mathrm{X}$ axis (Figure $7 \mathrm{~b}$ ).

4. The same happens with the third point (Figure 7c).

5. Lastly, the user clicks in the button again to confirm that the referential is correctly set and the cube appears at the defined origin with the specified orientation.

After the coordinate frame definition, the above mentioned rigid body was fixed in the controller (Figure $6 \mathrm{~b}$ ), and the system was properly set to begin tracking.

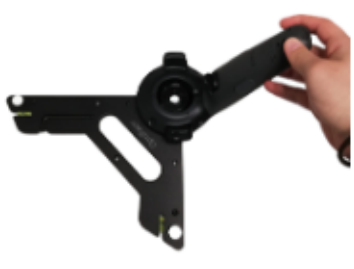

(a)

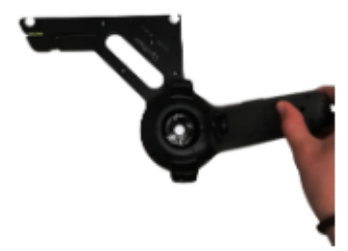

(b)

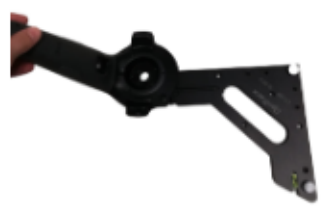

(c)

Figure 7. Referential definition on the HTC Vive application: (a) Step 1. (b) Step 2. (c) Step 3.

The data streaming was done identically as in HoloLens 2, using the ROS\# library developed by Siemens.

\subsection{Data Synchronization}

There were performed several tests, which can be divided into two categories: the ones with motion and the ones without motion. The data synchronization for the tests without motion were made using the ROS library message filters ${ }^{3}$. This filter subscribes to both topics (from the OptiTrack data and from the HL2 or HTC data) and synchronizes them accordingly to their timestamp that is included in the header. The policy used 
for synchronization was ApproximateTime because the rates of sampling were different, therefore the timestamps could not be directly matched. The data was then exported to a csv file for further analysis.

From the data acquired it was noted that the timestamp from HoloLens 2 had a small delay (less than one second). So, for the tests that involved movement, the synchronization through timestamp would not work. For this reason, the tracking data was saved into a cvs file, and synchronization performed a posteriori in Matlab. Figure 8 shows the signals representation of the OptiTrack and HoloLens 2 position (the represented position is referring to the $\mathrm{z}$ axis, as the subject only moved in that direction). To synchronize the data, the method used was to find the peaks (Figure 9a, maximum and minimum), and then, calculate the difference between the corresponding points in the temporal axis (horizontal). Then, the delay calculated was the mean of those differences. According to the delay calculated, the signals were readjusted (Figure 9b), and then, the accuracy was calculated. In the graph, it is also possible to verify some differences in the vertical axis between both signals, which indicates some errors in the position measures, as it will be discussed in section 4 .

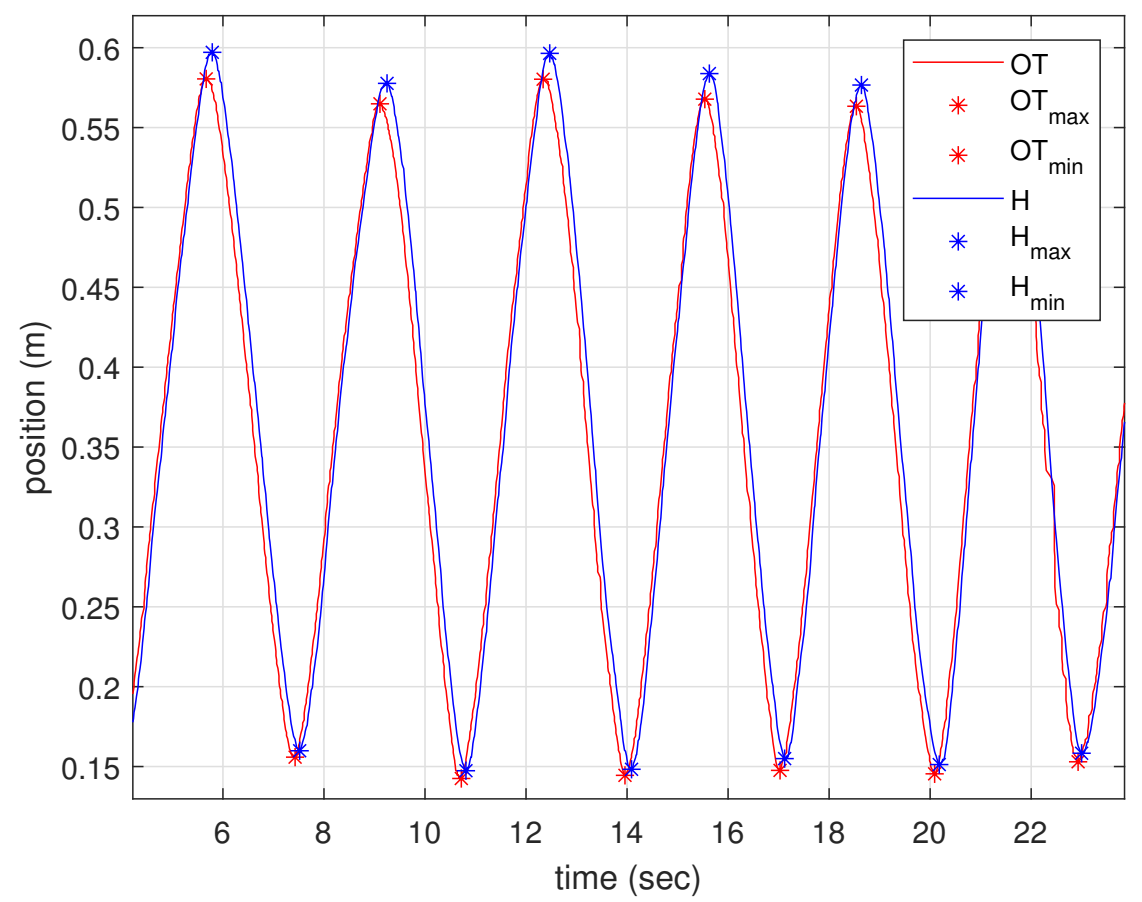

Figure 8. Position signals of OptiTrack (red) and HoloLens 2 (blue). 


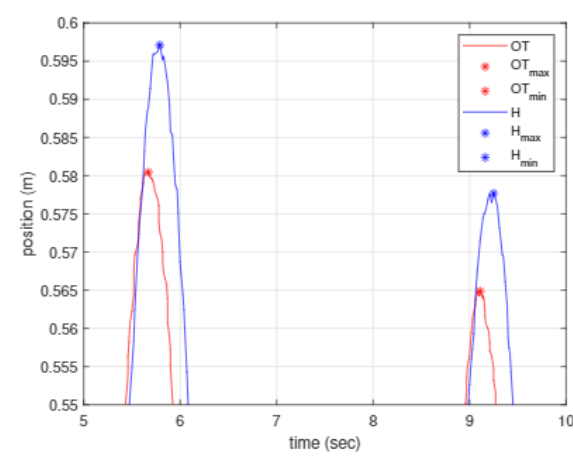

(a)

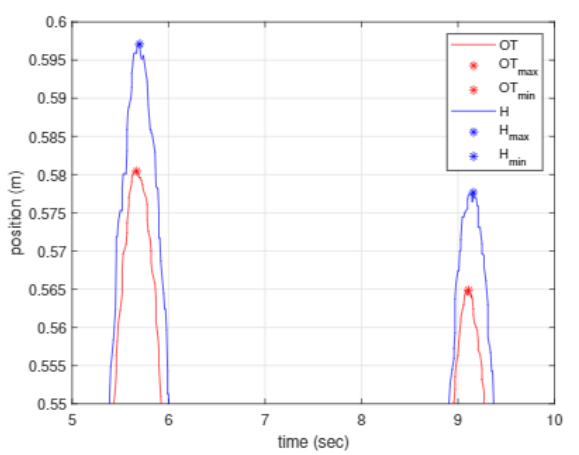

(b)

Figure 9. Comparison between original and synchronized data: (a) Original data. (b) Synchronized data.

\subsection{Data Analysis}

The data analysis was performed using MATLAB. For the tests without motion, the algorithm calculated the accuracy and the repeatability of each test.

The accuracy calculates the difference between the measured coordinates $\left(x_{H}, y_{H}, z_{H}\right)$ from HL2/HTC and the ground truth (measures from OptiTrack: $\left.x_{O T}, y_{O T}, z_{O T}\right)$. The equations to calculate the accuracy were based in ISO 9283 [11] and are represented in (1). Where $n$ represents the number of samples, and $e x, e y$ and $e z$ refer to the coordinates errors in the reference frame.

$$
A_{s}=\frac{1}{n} \sum_{i=1}^{n} \sqrt{e x_{i}^{2}+e y_{i}^{2}+e z_{i}^{2}}
$$

where,

$$
\begin{aligned}
& e x=x_{H}-x_{O T} \\
& e y=y_{H}-y_{O T} \\
& e z=z_{H}-z_{O T}
\end{aligned}
$$

According to ISO 9283, the repeatability $\left(R_{S}\right)$, also called precision, determines the variance of the measured points and it is calculated using distance between the measured values (from HL2/HTC) and their mean value $(\bar{l})$, and the standard deviation $\left(\sigma_{l}\right)$, as shown in (3). $\bar{x}, \bar{y}, \bar{z}$ are the mean of the measures of each axis, and $x_{i}, y_{i}, z_{i}$ are the measures of each axis in sample $i[8]$.

$$
R_{s}=\bar{l}+3 \sigma_{l}
$$

where,

$$
\begin{gathered}
\bar{l}=\frac{1}{n} \sum_{i=1}^{n} l_{i} \\
l_{i}=\sqrt{\left(x_{i}-\bar{x}\right)^{2}+\left(y_{i}-\bar{y}\right)^{2}+\left(z_{i}-\bar{z}\right)^{2}} \\
\sigma_{l}=\sqrt{\frac{\sum_{i=1}^{n}\left(l_{i}-\bar{l}\right)^{2}}{n-1}}
\end{gathered}
$$

For the experiments that required motion of the user's hand, the MATLAB algorithm first performed a synchronization, considering the time origin as the moment that ROS started receiving data and counting the time from that point. Because the sampling rate of OptiTrack was higher than HL2 and HTC, it is necessary to perform a data interpolation in these last two datasets. After the interpolation, both datasets are properly synchronized, have the same length, and it is possible to start the data analysis. 
The data analysis for the motion experiments was focused on the accuracy (as in the previous tests) and delay calculation. It was verified that in HoloLens 2 the device had a small delay detecting the hand movement: the hand's hologram delay can be seen while moving the hand. Therefore, the algorithm calculated first the delay, readjusts the vectors and then calculates the accuracy.

\subsection{Experiments}

The purpose of this study was to analyse the accuracy and repeatability of the HoloLens 2 hand tracking and the HTC Vive controller tracking. Therefore, several experiments were conducted to evaluate the tracking system's in different situations:

- When the tracking object was stationary;

- When the tracking object was moving at different velocities;

- When the HTC Vive system was measuring the controller's position with only one base station;

- When using HL2 the user was always moving his/her head around;

- When measuring the HL2 hand tracking outside the center of the field vision;

- When tracking hands from different people (different hand size and shape);

- When using the left hand to track instead of the right.

Tables 1 and 2 describe in detail the objectives and the conditions of the experiments performed in HTC Vive and HoloLens 2, respectively.

Table 1. HTC Vive Experiments.

\begin{tabular}{|c|c|c|}
\hline$\#$ & Objective & Conditions \\
\hline 1 & $\begin{array}{l}\text { Measure accuracy and repeatability } \\
\text { without motion }\end{array}$ & $\begin{array}{l}\text { Controller always in the same posi- } \\
\text { tion (on top of a table) }\end{array}$ \\
\hline 2 & $\begin{array}{l}\text { Analyse the influence of the base sta- } \\
\text { tions when working individually }\end{array}$ & $\begin{array}{l}\text { Controller in the same position (as } \\
\text { in \#1) with only one base station }\end{array}$ \\
\hline 3 & $\begin{array}{l}\text { Measure the influence of the orien- } \\
\text { tation of the controller in relation to } \\
\text { the base stations }\end{array}$ & $\begin{array}{l}\text { With the controller always on the } \\
\text { same spot, rotate it } 45^{\circ} \text { at a time until } \\
\text { it reaches the starting point }\end{array}$ \\
\hline $4-6$ & $\begin{array}{l}\text { Analyse the system's accuracy when } \\
\text { the controller is moving }\end{array}$ & $\begin{array}{l}\text { Controller moving at low, medium } \\
\text { and fast speeds (average of } 9,16,29 \\
\mathrm{~cm} / \mathrm{s} \text {, respectively) }\end{array}$ \\
\hline
\end{tabular}

Table 2. HoloLens 2 Experiments.

\begin{tabular}{|c|c|c|}
\hline \# & Objective & Conditions \\
\hline 7 & $\begin{array}{l}\text { Measure accuracy and repeatability } \\
\text { without hand motion }\end{array}$ & $\begin{array}{l}\text { Right hand in the same position (at } \\
\text { the center of the vision field) }\end{array}$ \\
\hline 8 & $\begin{array}{l}\text { Analyse the influence on the mea- } \\
\text { sures when moving the head }\end{array}$ & $\begin{array}{l}\text { Right hand in the same position and } \\
\text { move the head constantly in various } \\
\text { directions }\end{array}$ \\
\hline $9-12$ & $\begin{array}{l}\text { Measure the influence of hand track- } \\
\text { ing at the vertices of the projection's } \\
\text { vision field }\end{array}$ & $\begin{array}{l}\text { Right hand in the four corners of the } \\
\text { projection's vision field (no move- } \\
\text { ment) }\end{array}$ \\
\hline $13-15$ & $\begin{array}{l}\text { Analyse the system's accuracy when } \\
\text { the hand is moving }\end{array}$ & $\begin{array}{l}\text { Right hand moving at low, medium } \\
\text { and fast speeds (average of } 7,13,27 \\
\mathrm{~cm} / \mathrm{s} \text {, respectively) }\end{array}$ \\
\hline 16,17 & $\begin{array}{l}\text { Analyse the influence of different } \\
\text { hand sizes and shapes }\end{array}$ & $\begin{array}{l}\text { Right hand in the same position }(\# 16) \\
\text { and moving (\#17) but from different } \\
\text { people }\end{array}$ \\
\hline 18,19 & $\begin{array}{l}\text { Analyse the influence of right and } \\
\text { left hands }\end{array}$ & $\begin{array}{l}\text { Left hand in the same position (\#18), } \\
\text { left hand moving (\#19) }\end{array}$ \\
\hline
\end{tabular}




\section{Results and Discussion}

This section presents the results obtained with the experiments performed and a discussion is elaborated in order to draw conclusions. First there are presented the results for HTC Vive and then for Microsoft HoloLens 2.

\subsection{HTC Vive}

The results achieved in the experiments for HTC Vive were quite satisfactory (Figure 10). For the stationary experiment (\#1), the accuracy obtained was of $3.5 \mathrm{~mm}$ and the repeatability of $2.5 \mathrm{~mm}$. When the measures were being acquired by only one base station (\#2), it was verified that the error increased significantly (in Figure 10a this experiment is divided in 2_a and 2_b which represent the use of base station $A$ and $B$, respectively). For one of the base stations, it increased almost by more than six times in accuracy (resulting in $23.76 \mathrm{~mm})$, but the increase in repeatability was not significant $(1.84 \mathrm{~mm})$. On the other hand, when the same experiment was done by the other base station, the accuracy only increased by 2 times $(7.31 \mathrm{~mm})$, but the repeatability was almost 4 times higher $(8.10 \mathrm{~mm})$. The deterioration of the results agreed with our expectations, as HTC Vive was designed to be used with both base stations working simultaneously. These tests were performed in order to discover what the system's reaction would be in case of a temporary occlusion of one of the base stations. The results from experiment \#3 (Figure 10b) which measured the influence of the controller's angle with the base stations, were also expectable. It was verified that indeed some variation was noted not only in the accuracy but also in the repeatability. Nevertheless, the maximum variation observed in both was around $3 \mathrm{~mm}$. From these results, it can be concluded that the controller's orientation has some influence in the errors, but it is not significant. The different orientations were allocated through a full rotation of the hand controller, turning $45^{\circ}$ each time until reaching the $360^{\circ}$, in total there were 8 different orientations and always the same position.

In the experiments with motion (\#4,\#5,\#6), the accuracy obtained was worse than when the controller was stationary, but the variation was not linear, i.e., when the velocity increased the error did not increase accordingly. This set of experiments was done more than one time and the results were quite inconsistent. For example, sometimes the test with medium velocity had the best accuracy $(5.6 \mathrm{~mm})$ while in other experiment sets the worst $(13.4 \mathrm{~mm})$. In all the velocity tests performed, it was verified that the HTC Vive system does not have a significant delay in the measures acquisition when compared to the OptiTrack.

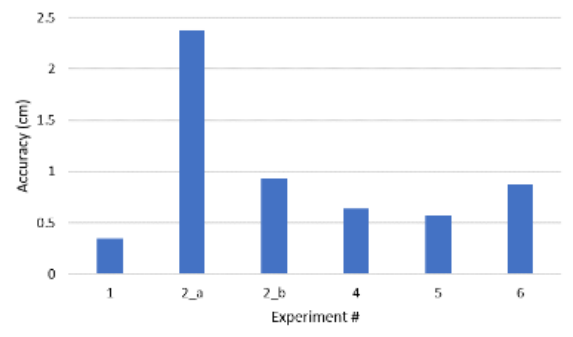

(a)

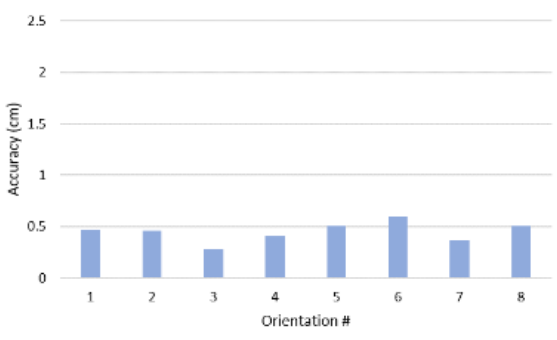

(b)

Figure 10. Accuracy graphs for all of the experiments performed on HTC Vive: (a) HTC Vive accuracy results. (b) Experiment \#3 accuracy results.

\subsection{Microsoft HoloLens 2}

The results obtained with HoloLens 2 were significantly worse in comparison with the HTC Vive (Figure 11). But it was expected, since HL2 depends on an algorithm to recognize the hand while the HTC Vive uses the inherited system controller with two base stations acquiring the infrared signals emitted by the controllers. For the experiment \#7, right hand stopped in the center of vision field, the accuracy obtained was around $18.3 \mathrm{~mm}$ and a 
repeatability of $5.8 \mathrm{~mm}$. While using the device, it is possible to verify that the hologram of the user's hand is not exactly aligned with the hand and it has some variation, turning out to be consistent with the results obtained. To analyse the influence of moving the head while tracking the hand, experiment \#8 revealed a small improvement in the accuracy of about $13 \%$ and the repeatability doubled. Therefore, the main conclusion retrieved from these experiments was that the head movement has some influence on the measures but not significantly enough so that it would be mandatory to have the head completely still during experiments.

The experiments \#9 to \#12 confirmed the supposition that errors are lower when the hand is positioned in center of the field of vision. The accuracy of the four experiments (corresponding to the four corners of the vision field) was between 23.9 and $26.3 \mathrm{~mm}$, and the repeatability between 5.9 and $7.2 \mathrm{~mm}$. These results point out that the hand's place in relation to the projection vision field may influence the measures accuracy and repeatability.

The velocity experiments (experiments \#13,\#14,\#15) had a delay of 65 milliseconds on average, which is observable while using the glasses: the hand's hologram is behind the real hand when moving. The accuracy estimated in these motion experiments are also not linear in response to speed variations. In fact, the results did not had a significant change, being around the $30 \mathrm{~mm}$ for the three velocities.

To analyse the influence of different hand sizes and shapes, experiments \#16 and \#17 were conducted with six different volunteers. The results obtained were quite interesting (Figure 11b), observing a significant variation in the accuracy results. The best one had an accuracy around $10 \mathrm{~mm}$, while the worst had an accuracy around $37.5 \mathrm{~mm}$. Reaching the conclusion that the hand size and shape have an interference in the measures, the person that performed the best experiment had the biggest hand of the participants, and the worst accuracy result's hand was the smallest. The motion experiments confirmed this conclusion, verifying also a difference of about $25 \mathrm{~mm}$ between the two extreme results.

Finally, experiments \#18 and \#19 showed some difference, although not significant, between the right and left hand (about $2.5 \mathrm{~mm}$ in accuracy when the hand was stopped), being the right hand the best result. In contrast to that result, in the movement experiment, the left hand presented the best result (20.9 $\mathrm{mm}$ against $31.2 \mathrm{~mm}$ of the right hand). In conclusion that right and left hands can have a small influence in the results, but it is neither linear nor predictable.

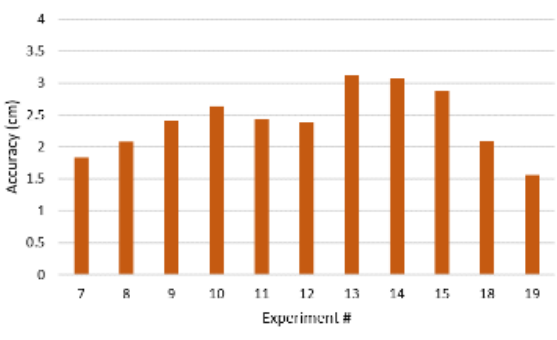

(a)

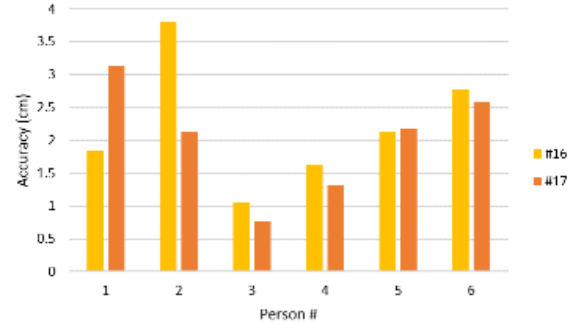

(b)

Figure 11. Accuracy graphs for all of the experiments performed on HoloLens 2: (a) HoloLens 2 accuracy results. (b) Experiments \#16-17 results.

\section{Conclusions}

This study presented the study of accuracy and repeatability in HoloLens 2 and HTC Vive systems in comparison with OptiTrack system that was considered as reference. In the first one, the method used was hand tracking, while in the second one the object tracked was its controller. After performing a series of tests, it was concluded that both devices show great potential for a vast number of applications in various area fields. However, 
HTC Vive presented better performance results, indicating that it would be more suitable than HoloLens 2 for applications that require high accuracy.

As a general rule, it can be concluded that HoloLens 2 would be more suitable for tasks that would not require high accuracy to achieve a good performance such as detection of intrusion in security areas, gesture recognition, and some painting applications. HTC Vive, on the other hand, would be also suitable for applications higher accuracy, such as tighten a screw on a car engine or arc welding metal pieces.

HTC Vive has already been used to improve the learning experience of medical students [12], and in a rehabilitation training program for upper limbs, where the patient would manipulate the controller according to the task requirements [13]. Flueratoru et al. [14] also claimed that HTC Vive could be used to acquire baseline measurements for the Ultra-wide-band system, whose accuracy and precision are in the range of centimeters. Kharvari and Hohl [15] tested the hypothesis of using VR in architectural education for studying precedents and found that it motivated the students to deepen their learning on the subject because of its interactivity.

As for HoloLens 2, it has already been used in industry to program industrial robots by demonstration, as it was mentioned in Section 2. But it is important to notice that not robot applications would be suitable for this type of programming due to its limited accuracy. For example, it could be suitable for a pick and place application. Sharma et al. [16] were able to improve building evacuation time and eradicate injuries and fatalities during emergencies, thanks to a HoloLens application that provided visual representation of a building on campus in 3D space. HoloLens has also entered the field of Nuclear Power Engineering helping maintenance workers get tasks done faster by providing them with content of plant layout and key equipment as holographic images [17].

Lastly, this study was a significant step and the base of the on going project of developing a human-robot interface to program by demonstration an industrial robot using Augmented Reality.

Author Contributions: Conceptualization, I.S., M.P. and A.M.; methodology, I.S.; software, I.S.; validation, I.S., R.S., M.P. and A.M.; investigation, I.S.; writing—original draft preparation, I.S.; writing - review and editing, I.S., R.S., M.P. and A.M.; supervision, M.P. and A.M.; funding acquisition, M.P. and A.M.. All authors have read and agreed to the published version of the manuscript.

Funding: Work realized within the scope of the project PRODUTECH4SC - POCI-01-0247-FEDER046102, funded by the ERDF - European Regional Development Fund, through the Operational Programme for Competitiveness and Internationalisation - COMPETE 2020.

Conflicts of Interest: The authors declare no conflict of interest.

\section{Abbreviations}

The following abbreviations are used in this manuscript:

$\begin{array}{ll}\text { AR } & \text { Augmented Reality } \\ \text { HL2 } & \text { HoloLens 2 } \\ \text { MR } & \text { Mixed Reality } \\ \text { MRTK } & \text { Mixed Reality Toolkit } \\ \text { ROS } & \text { Robot Operating System } \\ \text { VR } & \text { Virtual Reality } \\ \text { XR } & \text { Extended Reality }\end{array}$

\section{References}

1. Fang, H.C.; Ong, S.K.; Nee, A.Y. A novel augmented reality-based interface for robot path planning. International Journal on Interactive Design and Manufacturing 2014. doi:10.1007/s12008-013-0191-2.

2. Gironacci, I.; Vincs, K.; Mccormick, J. A Recommender System of Extended Reality Experiences. ACM International Conference Proceeding Series, 2020. doi:10.1145/3383812.3383839.

3. De Pace, F.; Manuri, F.; Sanna, A. Augmented Reality in Industry 4.0. American Journal of Computer Science and Information Technology 2018, 06, 0-7. doi:10.21767/2349-3917.100017. 
4. Rahul, K.; Raj, V.P.; Srinivasan, K.; Deepa, N.; Kumar, N.S. A Study on Virtual and Augmented Reality in Real-Time Surgery. 2019 IEEE International Conference on Consumer Electronics - Taiwan, ICCE-TW 2019, 2019. doi:10.1109/ICCE-TW46550.2019.8992009.

5. Ullah, F.; Sepasgozar, S.M.; Wang, C. A systematic review of smart real estate technology: Drivers of, and barriers to, the use of digital disruptive technologies and online platforms, 2018. doi:10.3390/su10093142.

6. Alhamdan, Y.; Alabachi, S.; Khan, N. Extended Abstract: CoShopper - Leveraging Artificial Intelligence for an Enhanced Augmented Reality Grocery Shopping Experience. Proceedings - 2020 IEEE International Conference on Artificial Intelligence and Virtual Reality, AIVR 2020, 2020. doi:10.1109/AIVR50618.2020.00069.

7. Rudorfer, M.; Guhl, J.; Hoffmann, P.; Kruger, J. Holo Pick'n'Place. IEEE International Conference on Emerging Technologies and Factory Automation, ETFA, 2018. doi:10.1109/ETFA.2018.8502527.

8. Blankemeyer, S.; Wiemann, R.; Posniak, L.; Pregizer, C.; Raatz, A. Intuitive robot programming using augmented reality. Procedia CIRP, 2018. doi:10.1016/j.procir.2018.02.028.

9. Spitzley, K.A.; Karduna, A.R. Feasibility of using a fully immersive virtual reality system for kinematic data collection. Journal of Biomechanics 2019. doi:10.1016/j.jbiomech.2019.02.015.

10. Niehorster, D.C.; Li, L.; Lappe, M. The accuracy and precision of position and orientation tracking in the HTC vive virtual reality system for scientific research. i-Perception 2017. doi:10.1177/2041669517708205.

11. ISO 9283:1998 - Manipulating industrial robots - Performance criteria and related test methods. Standard, ISO/TC 299 Robotics, 1998.

12. Gonzalez, D.C.; Garnique, L.V. Development of a Simulator with HTC Vive Using Gamification to Improve the Learning Experience in Medical Students. 2018 Congreso Internacional de Innovacion y Tendencias en Ingenieria, CONIITI 2018 Proceedings, 2018. doi:10.1109/CONIITI.2018.8587058.

13. Chen, D.; Liu, H.; Ren, Z. Application of Wearable Device HTC VIVE in Upper Limb Rehabilitation Training. Proceedings of 2018 2nd IEEE Advanced Information Management, Communicates, Electronic and Automation Control Conference, IMCEC 2018, 2018. doi:10.1109/IMCEC.2018.8469540.

14. Flueratoru, L.; Simona Lohan, E.; Nurmi, J.; Niculescu, D. HTC Vive as a Ground-Truth System for Anchor-Based Indoor Localization. International Congress on Ultra Modern Telecommunications and Control Systems and Workshops, 2020. doi:10.1109/ICUMT51630.2020.9222439.

15. Kharvari, F.; Hohl, W. The role of serious gaming using virtual reality applications for 3D architectural visualization. 2019 11th International Conference on Virtual Worlds and Games for Serious Applications, VS-Games 2019 - Proceedings, 2019. doi:10.1109/VS-Games.2019.8864576.

16. Sharma, S.; Bodempudi, S.T.; Scribner, D. Identifying Anomalous Behavior in a Building Using HoloLens for Emergency Response. IS and T International Symposium on Electronic Imaging Science and Technology, 2020. doi:10.2352/ISSN.24701173.2020.13.ERVR-224.

17. Zhang, Y.; Li, D.; Wang, H.; Yang, Z.H. Application of Mixed Reality Based on Hololens in Nuclear Power Engineering. Lecture Notes in Electrical Engineering, 2020. doi:10.1007/978-981-15-1876-8\_2. 